\title{
Solitary metastasis of urothelial carcinoma of the urinary bladder to the heart: An unusual clinical manifestation
}

\author{
Konstantinos Spiliotopoulos, MD, ${ }^{\mathrm{a}}$ Mihalis Argiriou, MD, FETCS, ${ }^{\mathrm{a}}$ Theodoros Argyrakos, MD, ${ }^{\mathrm{b}}$ Konstantinos Haritopoulos, PhD, \\ FEBU, ${ }^{\mathrm{d}}$ Konstantinos Kanakakis, MD, ${ }^{\mathrm{a}}$ Timotheos Sakellaridis, MD, ${ }^{\mathrm{a}}$ Christos Charitos, MD, ${ }^{\mathrm{a}}$ and Ion Bellenis, MD, PhD, FETCS, \\ FRCS, ${ }^{\mathrm{c}}$ Athens, Greece
}

\section{Supplemental material is available online.}

Approximately $10 \%$ of metastatic tumors eventually reach the heart or pericardium, and almost every type of malignant tumor has been known to do so. Secondary neoplasms are 20 to 40 times more common than primary cardiac malignancies. In an effort to define better the broad spectrum of the metastatic tumors reaching the heart and their diverse clinical behavior, we present this rare case of a symptomatic, isolated urothelial carcinoma of the bladder metastatic to the heart.

\section{CLINICAL SUMMARY}

A 66-year-old man was referred to our department for a large pericardial effusion and cardiac tamponade. He also had a nonproductive cough and a worsening progressive dyspnea of several days' duration. His medical history included a radical cystectomy and urinary diversion (ileal conduit after Bricker procedure) performed 5 years previously for a high-grade infiltrating urothelial carcinoma (World Health Organization classification) of the urinary bladder (T2N0M0), with negative results of follow-up since then.

The echocardiogram revealed a multilobular mass attached to the wall of the right atrium with no blood flow in it, extending beyond the tricuspid valve annulus and compromising its function. A subxiphoid pericardiocentesis drained approximately $2000 \mathrm{~mL}$ of bloody fluid, the cytologic examination of which showed many mesothelial cells and other cells with uncertain morphologic features. After the patient's condition was stabilized, he underwent chest computed tomography and magnetic resonance imaging, which revealed an enhancing cardiac mass involving the

\footnotetext{
From the Departments of Cardiac Surgery, ${ }^{\mathrm{a}}$ Pathology, ${ }^{\mathrm{b}}$ and Thoracic and Vascular Surgery, ${ }^{\mathrm{c}}$ Evangelismos General Hospital, and the Department of Urology, Attikon University Hospital, ${ }^{\mathrm{d}}$ Athens, Greece.

Received for publication Nov 12, 2007; accepted for publication Nov 26, 2007. Address for reprints: Konstantinos Spiliotopoulos, MD, Haritos 31, 106 75, Kolonaki, Athens, Greece (E-mail: cvspiliotopoulos@hotmail.com).

J Thorac Cardiovasc Surg 2008;136:1377-8

$0022-5223 / \$ 34.00$

Copyright (c) 2008 by The American Association for Thoracic Surgery doi:10.1016/j.jtcvs.2007.11.046
}

entire right side of the heart, from the right atrial appendage to the right ventricle and into the origin of the pulmonary artery (Figure 2A). A full-body workup followed, including bone scan and computed tomographic scans of the head, chest, abdomen, and pelvis, all of which yielded negative results for other metastatic sites.

Transvenous endomyocardial biopsy of this mass was performed. Histologic examination of the specimen showed infiltration of the myocardium by an undifferentiated neoplasm formed by epithelioid cells with eosinophilic cytoplasm and eccentric atypical nuclei with signet ring-like morphology (Figure 1A). Histochemically, the cells stained positively with periodic acid-Schiff and negatively with Alcian blue. Immunohistochemically, they expressed cytokeratin 7, cytokeratin AE1, epithelial membrane antigen, the anti-adhesion molecules mucins 1 and 2, vimentin, and p63 (Figure E1). They did not express uroplakin, CD56, synaptophysin, chromogranin, the myogenic differentiation transcription factor 1, desmin, smooth muscle actin, CD34, CD117, thyroid transcription factor 1, thrombomodulin, or calretinin. The reevaluation of the histologic slides from the patient's cystectomy specimen showed the same neoplasm infiltrating the urinary bladder (Figure 1B); the neoplasm in the myocardium was therefore considered to be metastatic urothelial (transitional cell) carcinoma.

After this diagnosis the patient followed a chemotherapy regimen based on carboplatin and gemcitabine, with a $40 \%$ reduction of the size of the cardiac mass and a reduction of the pericardial effusion seen on 6-month follow-up chest magnetic resonance imaging (Figure 2B). A year after the original diagnosis, the patient is free of symptoms and has no sign of disease spread outside the heart. The size of the metastatic tumor has remained stable.

\section{DISCUSSION}

Bladder cancer is the fourth most common cancer among men and the tenth most common among women. Localized bladder cancer is classified as superficial disease, limited to the mucosa and lamina propria, or invasive disease, extending into the muscularis and beyond. Locoregional control with radical cystectomy offers the best opportunity for survival; administration of adjuvant chemotherapy to patients with organ-confined bladder cancer (stage T1 or T2) does not provide either a survival advantage or an improvement 

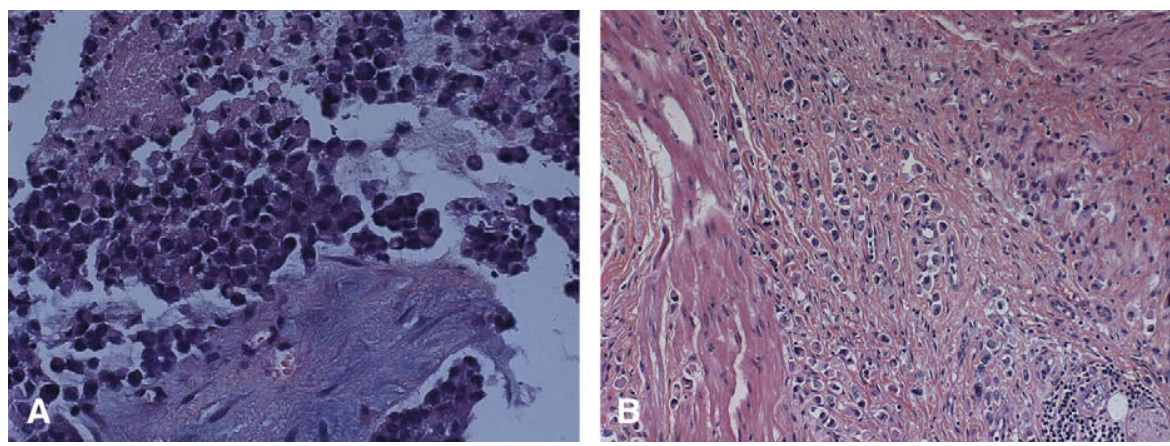

FIGURE 1. A, Photomicrograph of urothelial carcinoma metastatic to myocardium. B, Urothelial carcinoma infiltrating urinary bladder muscular wall (primary tumor). (Hematoxylin-eosin stain, original magnification $40 \times$ ).
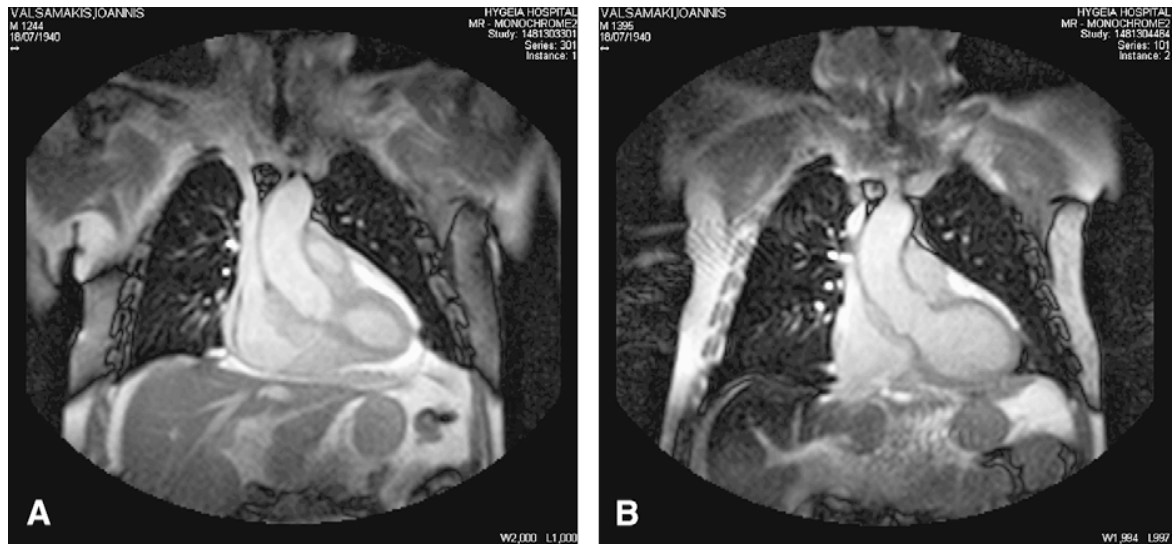

FIGURE 2. Coronal magnetic resonance images demonstrating metastatic tumor involving entire right side of heart and infiltrating myocardium before (A) and after (B) chemotherapy resulting in $40 \%$ reduction in size.

in local control after cystectomy. Dissemination of urothelial carcinoma of the bladder generally follows predictable patterns, first involving regional and juxtaregional lymph nodes, then spreading to the liver, lungs, and bones and, less commonly, involving the intestine, adrenal glands and kidneys. ${ }^{1}$

This case presents uncommon features. Isolated metastasis of bladder tumors to the heart is extremely rare, with only 2 other reported cases. ${ }^{2}$ Such a long survival-more than a year after diagnosis of the metastatic disease-has never been reported in any similar case to our knowledge, with survival among patients with cardiac metastases known to be uniformly poor. Finally, clinical manifestations were present, although cardiac metastases are usually clinically silent and rarely produce symptoms, the most common being pericardial effusion or cardiac tamponade. ${ }^{3,4}$

Because of the advanced stage at diagnosis, curative surgery is only possible for a minority of patients with cardiac metastatic tumors, especially when this is the only site of dis- ease and the primary tumor is under control or when metastases result in right ventricular outflow tract obstruction. ${ }^{4,5}$ In this setting, surgical debulking may ameliorate symptoms and prolong survival, as may chemotherapy, which our patient elected. Otherwise, surgical therapy is usually limited to relief of recurrent pericardial effusions or tamponade, usually through subxiphoid pericardiotomy.

\section{References}

1. Schoenberg M. Management of invasive and metastatic bladder cancer. In: Walsh PC, Retic AB, Vaughan ED Jr., Wein AJ, eds. Campbell's urology, vol 4. 8th ed. Philadelphia: Saunders; 2002. p. 2803-13.

2. Stricker PD, Grant AB. Solitary metastasis of squamous cell carcinoma of the bladder to the myocardium. J Urol. 1987;137:113-4.

3. Malde DJ, Gall Z, George N. Ventricular rupture secondary to cardiac metastasis of transitional cell carcinoma of the bladder. Scand J Urol Nephrol. 2006;40: 170-1.

4. Clemente LM, Patier JL, Lopez-Suanzes MJ, Jimenez M. Cardiac metastases from a transitional cell carcinoma: an unusual clinical manifestation. Br J Urol. 1997;80: 831-2.

5. Kemp W, Rothberg M, Saporito JJ, Bergstrom I. Transitional cell carcinoma and right ventricular obstruction. J Urol. 1997;158:1522-3. 


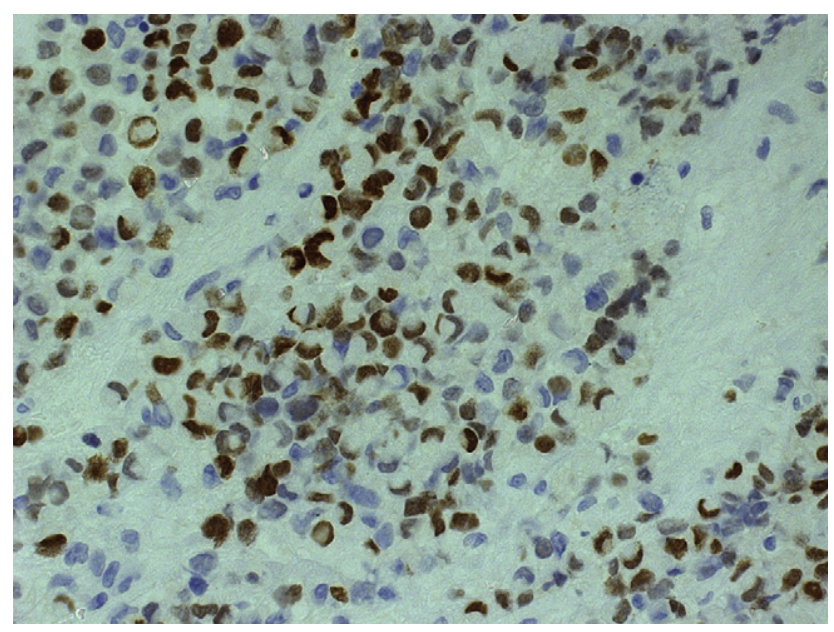

FIGURE E1. Nuclear expression of p63 in neoplastic cells infiltrating myocardium. (3,3'-diaminobenzidine, original magnification $40 \times)$. 